\title{
Preface
}

\section{An atlas of head and neck images, part II}

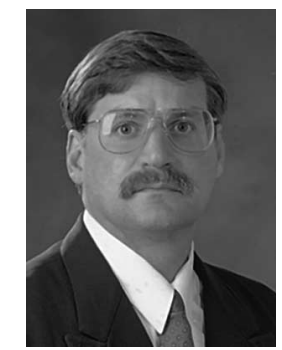

Richard H. Haug, DDS

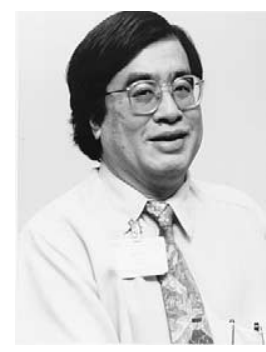

Charles Lee, MD

Guest Editors

Part I of An Atlas of Head and Neck Images reviewed imaging for the head and neck in trauma, chest pathology, and ultrasonography for the head and neck. It was offered both as an overview of technology and as a simple guide for the interpretation of images common to oral and maxillofacial surgeons, plastic and reconstructive surgeons, otorhinolaryngologists, and head and neck surgeons. It was also intended as a useful tool for radiologists, neurosurgeons, general surgeons, and the medical and dental communities at large.

Part II of our series begins with an exhaustive compendium of panoramic images. These are provided not only as a quick review of common and uncommon pathoses of the mandible or maxilla, but also as a visual reference for how these lesions are most likely to appear on panoramic radiographs. The next article focuses on computed tomography of the head and neck, providing representative images of the most common pathologic head and neck entities. An article on nuclear imaging provides a synopsis of the most frequently encountered vascular lesions of the head, face, and neck. Finally, an article on magnetic resonance imaging complements the two issues by providing one more additional imaging modality in the practitioner's diagnostic armamentarium.

Although this atlas series does provide an overview of signs, symptoms, etiology, and pathophysiology of a vast array of head and neck problems, it is not meant to be a definitive treatise on injury and pathology. It is intended merely to quickly point practicing surgeons in the right direction when specific conditions and problems in head and neck surgery are encountered. It is highly visual in design so that it may serve as a quick and ready reference.

Richard H. Haug, DDS

Division of Oral and Maxillofacial Surgery University of Kentucky College of Dentistry D-509 Chandler Medical Center Lexington, KY 40536-0297, USA

E-mail address: rhhaug@pop.uky.edu

Charles Lee, MD Division of Diagnostic Radiology University of Kentucky College of Medicine Chandler Medical Center Lexington, KY 40536-0297, USA

E-mail address: clee0@pop.uky.edu 